\title{
Blood Lysophosphatidylcholine (LPC) Levels and Characteristic Molecular Species in Neonates: Prolonged Low Blood LPC Levels in Very Low Birth Weight Infants
}

\author{
AKIHIRO TAKATERA, ATSUKO TAKEUCHI, KAYOKO SAIKI, ICHIRO MORIOKA, NAOKI YOKOYAMA, \\ AND MASAFUMI MATSUO
}

Department of Pediatrics [A.T., K.S., I.M., N.Y., M.M.], Kobe University Graduate School of Medicine, Kobe 650-0017, Japan; Division of Analytical Laboratory [A.T.], Kobe Pharmaceutical University, Kobe 658-8558, Japan

\begin{abstract}
Lysophosphatidylcholine (LPC) has various stimulatory effects on many types of immune cells. The purpose of our study was to characterize blood LPC levels and to determine the composition of LPC molecular species (LPCs) in the neonatal period. Thirty-six neonates were enrolled in this study and then grouped according to birth-weight as follows: non-very low birth weight (NVLBW); $\geq 1,500 \mathrm{~g}(n=17)$, and very low birth weight (VLBW); $<1,500 \mathrm{~g}(n=19)$. Sixteen healthy normal adults were used as controls. Levels of total blood LPC and LPCs (16:0-, 18:0-, 18:1-, 18:2-, and 20:4-LPC species) were measured using HPLC coupled with tandem mass spectrometry. Total blood LPC levels at birth in neonates in both groups (NVLBW and VLBW) were significantly lower than those of adult levels. In NVLBW infants, LPC levels reached adult levels at postnatal day 3 compared with VLBW infants, who attained adult levels after postnatal day 57 (around full-term). The composition of the LPCs was different not only between neonates and adults, but between NVLBW and VLBW infants. These findings may be associated with the difference of immunity among adults, NVLBW, and VLBW infants. (Pediatr Res 62: 477-482, 2007)
\end{abstract}

$\mathrm{L}^{2}$ ysophosphatidylcholine (LPC) is one of the bioactive lysophospholipids, which has various stimulatory effects in many types of immune cells in vitro (1) such as monocytes (2), macrophages $(3,4)$, T lymphocytes $(5,6)$ and neutrophils (7). The lengths and saturation of their acyl chains distinguish molecular species of LPC (Fig. 1). Recent research has indicated that the main LPC molecular species (LPCs), i.e., 16:0-, 18:0-, 18:1- and 18:2-LPC, are markedly reduced in adult patients with sepsis, and there is an inverse correlation between the severity of infection and blood LPC levels (8). Other research has shown that administration of 18:0- or 18:1-LPC to mouse models of sepsis protect them against lethality (9). LPC may play an important role in human neonates as well.

Recently, we established a method for quantification of blood LPCs in neonates with birth weight more than $1,500 \mathrm{~g}$ (non-very low birth weight: NVLBW) (10). However, there is no data of blood LPC levels in very low birth weight (VLBW)

Received February 21, 2007; accepted May 27, 2007.

Correspondence: Ichiro Morioka, M.D., Ph.D., Department of Pediatrics, Kobe University Graduate School of Medicine, 7-5-1 Kusunokicho, Chuo-ku, Kobe 650-0017 Japan; e-mail: ichim@med.kobe-u.ac.jp

This work was supported by a Grant-in-Aid for Scientific Research from the Morinaga Hoshi-kai Foundation. infants. Because VLBW infants are more susceptible to invasion by pathogenic microbes than NVLBW infants, we hypothesized that one of the causes of susceptibility to infection is due to prolonged lower blood LPCs level in the neonatal period of VLBW infants. In addition, the relationship between blood LPC levels in neonates and nourishment has not been reported, although LPC is an element included in human breast milk (11). We hypothesized that feeding influences the increase in blood LPC levels after birth.

The purpose of our study was to detect blood LPCs levels not only in NVLBW infants, but also in VLBW infants and to clarify the correlation between blood LPCs levels and the amount of feeding in the neonatal period.

\section{MATERIALS AND METHODS}

Subjects. With the approval of the Ethics Committee of Kobe University Graduate School of Medicine, 36 newborn infants without infection, who were admitted to our neonatal intensive care unit (NICU) in the Kobe University Hospital, Kobe, Japan, from April 2004 to March 2006, were enrolled into the present study. Their bloods were sampled after informed consent had been obtained from their parents.

Sample preparation. The blood was sampled from the venous vein, and was centrifuged to separate the serum. The blood samples were obtained at postnatal day $0,1,2,3-4,5-7,8-14,15-21,22-28,29-56$, and after day 57 . The specimens were stored at $-20^{\circ} \mathrm{C}$ until assay. The adult blood was obtained by the same procedure described above from 16 healthy normal adults.

LPC measurements. The main LPCs, such as 16:0-, 18:0-, 18:1-, 18:2-, and 20:4-LPC were measured in this study, and total LPC levels were regarded as the sum of these LPCs. The blood LPCs levels were measured by HPLC coupled with tandem mass spectrometry (LC-MS/MS) as previously established (10). Briefly, to $20 \mu \mathrm{L}$ of the serum sample, we added $100 \mu \mathrm{L}$ of methanol solution with internal standards (IS) 18:0-D35 LPC (2 $\mu \mathrm{g})$. Purification of phospholipids was performed using solid phase extraction from 20 $\mu \mathrm{L}$ of serum with an IS. Entire elute analytes were dried using a gentle stream of nitrogen. The residue was dissolved in $1 \mathrm{~mL}$ of methanol, and an aliquot of $15 \mu \mathrm{L}$ was injected into the LC-MS/MS system. A Shimadzu 10ADvp LC system (Shimadzu, Kyoto, Japan) was used to inject sample solutions on a Cosmosil $5 \mathrm{NH}_{2}$-MS column $(4.6$ i.d. $\times 50 \mathrm{~mm}, 5.0 \mu \mathrm{m})$ from Nacalai Tesque Inc. (Kyoto, Japan). The isocratic mobile phase, a mixture of $0.2 \%$ (vol/vol) formic acid in methanol, was delivered at $0.2 \mathrm{~mL} / \mathrm{min}$ into the mass spectrometer ionization chamber. Quantitation was achieved by MRM positive ion mode using an API 3000 mass spectrometer (Applied Biosystems / MDS Sciex, Toronto, Canada), equipped with a Turboionspray ${ }^{\mathrm{TM}}$ interface at $400^{\circ} \mathrm{C}$. Under the ESI optimized conditions, $[\mathrm{M}+\mathrm{H}]^{+}$ions of LPCs generated

Abbreviations: LPC, lysophosphatidylcholine; LPCs, lysophosphatidylcholine molecular species; NVLBW, non-very low birth weight; VLBW, very low birth weight 
<smiles>[R]C(=O)OCC(O)COP(=O)([O-])OCC[NH+]C</smiles>

\begin{tabular}{|c|c|}
\hline LPC species & \multicolumn{1}{|c|}{$\mathbf{R}$} \\
\hline $16: 0-\mathrm{LPC}$ & $-\mathrm{C}_{15} \mathrm{H}_{31}$ \\
18:0-LPC & $-\mathrm{C}_{17} \mathrm{H}_{35}$ \\
18:1-LPC & $-\mathrm{C}_{17} \mathrm{H}_{33}$ \\
18:2-LPC & $-\mathrm{C}_{17} \mathrm{H}_{31}$ \\
20:4-LPC & $-\mathrm{C}_{19} \mathrm{H}_{31}$ \\
\hline
\end{tabular}

Figure 1. Structural formula of the LPC molecular species: The lengths and saturation of their acyl chains distinguish the molecular species of LPC. We quantified 16:0-, 18:0-, 18:1-, 18:2-, and 20:4-LPC in this study.

ions at $\mathrm{m} / \mathrm{z} 184.2$ as the major product ion. Analytical data were processed by Analyst software 1.3.1.

Classification. Thirty-six infants without infection were grouped according to birth-weight as follows; NVLBW $(n=19)$ included infants whose birth weight was over 1,500 $\mathrm{g}$ and VLBW $(n=17)$ included infants less than 1,500 g. Characteristics were obtained by the medical records, and assessment of the relationship between these characters was conducted. The characteristics of the neonates, who were enrolled in this study, are shown in Table 1. Since the two groups (NVLBW and VLBW) were distinguished by birth weight, all characteristics except gender were significantly different between these groups. Sixteen healthy adult bloods were used as controls.

Calculations of the amount of feeding. The amount of feeding, including both breast feeding and non-breast breast feeding, of each infant was calculated at a specific day point and was shown as $\mathrm{ml} / \mathrm{kg} / \mathrm{d}$.

Statistical analysis. Statistical analyses were performed using Excel 2004 (Microsoft, USA) with add-in software Statcel version 2.0 (OMS, Tokyo, JAPAN). Results were expressed as the peak area ratio of each analyte to the IS (mean \pm SEM). Neonatal blood LPCs levels were assessed by comparison of each group. The unpaired $t$ test was used to determine statistical significance. Differences in infant characteristics were determined by $\chi^{2}$ for independence test. The relation between the neonatal LPC levels and the amount of feeding was investigated by Pearson's correlation coefficient test. In all tests, $p<0.05$ was considered significant.

\section{RESULTS}

The numbers of analyzed blood samples. The blood samples of NVLBW infants at postnatal day $0(n=16), 1(n=$

Table 1. Characteristics of the neonates

\begin{tabular}{lcc}
\hline & NVLBW $(\mathrm{n}=19)$ & VLBW $(\mathrm{n}=17)$ \\
\hline $\begin{array}{l}\text { Male (n) } \\
\text { Gestation (weeks) }\end{array}$ & 10 & 11 \\
$\begin{array}{l}\text { Birth weight (g) } \\
\text { Age of beginning of feeding } \\
\quad \text { days) }\end{array}$ & $2414(150-41)$ & $28(23-32)^{*}$ \\
$\begin{array}{l}\text { Age of reached full feeding } \\
\quad \text { (days) }\end{array}$ & $4(3-8)$ & $3(1-5))^{*}$ \\
\end{tabular}

Full feeding, $100 \mathrm{ml} / \mathrm{kg} /$ day. All values are given as medians (range). $* p<0.05$.
$11), 2(n=6), 3-4(n=18), 5-7(n=10), 8-14(n=3)$, $15-21(n=1)$, and of VLBW infants at postnatal day 0 $(n=5), 1(n=8), 2(n=8), 3-4(n=5), 5-7(n=4)$, $8-14(n=9), 15-21(n=13), 22-28(n=11), 29-56$ $(n=18), 57-(n=2)$, were analyzed.

Comparison of total blood LPC levels between neonates and the adult. Total blood LPC levels in the healthy adult were $2.87 \pm 0.19$ (peak area ratio to IS). In neonates, total blood LPC levels at day 0 were $1.06 \pm 0.06$ in NVLBW and $0.81 \pm 0.24$ in VLBW infants, with no significant difference between these two groups (Fig. 2). In these neonatal groups, total blood LPC levels at day 0 were significantly lower than adult levels. In NVLBW infants, blood LPC levels on days 3-4 reached $2.07 \pm 0.13$, and there was no significant difference compared with the adult levels. In VLBW infants, blood LPC levels increased slowly and reached $2.28 \pm 0.02$ after day 57 (Fig. 2). In addition, in VLBW infants, the neonatal blood LPC levels within 2 mo were significantly lower than the adult levels.

Comparison of total blood LPC levels between NVLBW and VLBW infants. Total blood LPC levels at days $0-1$ in both groups were similar. However, total blood LPC levels from day 2 to day 7 in VLBW infants were significantly lower than LPC levels in NVLBW infants. Total blood LPC levels in NVLBW infants reached $2.58 \pm 0.24$ at day 7 , but total blood LPC levels in VLBW infants were only $1.44 \pm 0.32$ by day 7 . There was no significant difference between the two groups

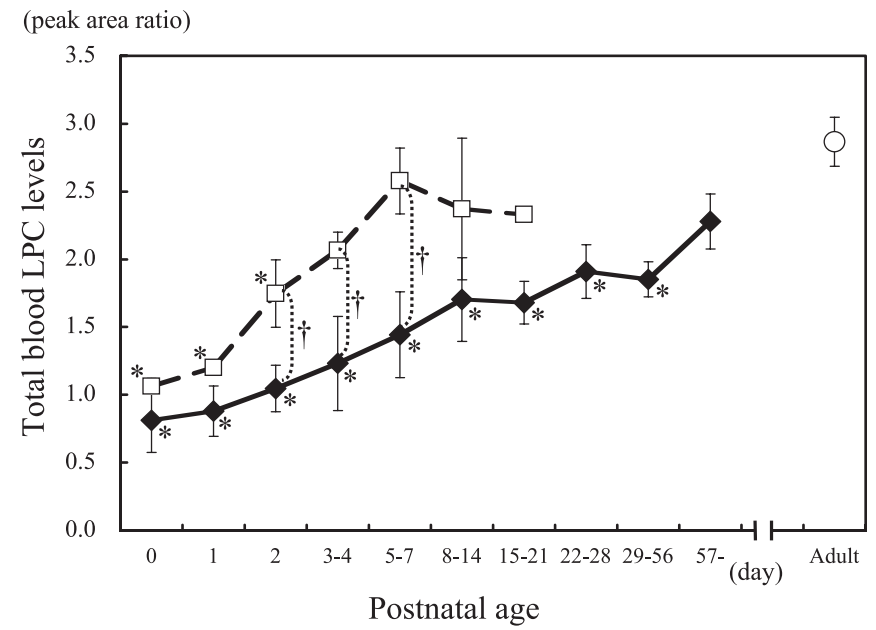

Figure 2. Chronological changes in blood LPC levels and comparison of these levels among NVLBW infants $(\square, n=19$, numbers of analyzed blood samples: day $0(n=16), 1(n=11), 2(n=6), 3-4(n=18), 5-7$ $(n=10), 8-14(n=3), 15-21(n=1))$, VLBW infants $(\diamond, n=17$, numbers of analyzed blood samples: day $0(n=5), 1(n=8), 2(n=8), 3-4(n=5)$, $5-7(n=4), 8-14(n=9), 15-21(n=13), 22-28(n=11), 29-56(n=18)$, after day $57(n=2))$, and adults $(\bigcirc, n=16)$. Blood LPC levels at birth were low in both NVLBW and VLBW infants in comparison to those in adults. However, in both groups, LPC levels increased each day. In VLBW infants, the increase in LPC levels was less than that in NVLBW infants. Therefore, in VLBW infants, LPC levels were significantly lower than those in NVLBW infants from day 2 to day 7. In NVLBW infants, LPC levels reached adult levels by day 3. On the other hand, in VLBW infants, LPC levels were significantly lower than those in adults until 2 mo old. LPC levels were expressed as the peak area ratio of each analyte to the IS (mean \pm SEM). ${ }^{*} p<0.05$ compared with adults; $\uparrow p<0.05$ NVLBW compared with VLBW infants. 

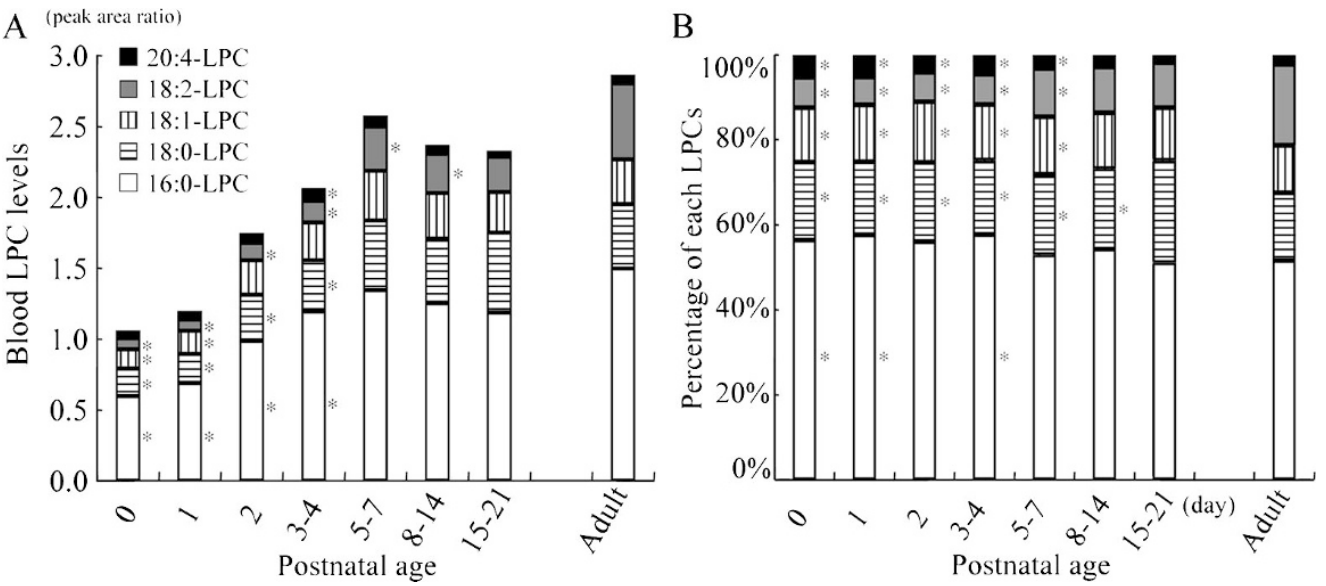

Figure 3. Change in LPCs in NVLBW infants: $(A)$ Change in blood LPC levels distinguished by molecular species. LPCs levels were expressed as the peak area ratio of each analyte to the IS (mean). ( $B$ ) Change in percentage of each LPCs. Postnatal day $0(n=16), 1(n=11), 2$ $(n=6), 3-4(n=18), 5-7(n=10)$, 8-14 $(n=3), 15-21(n=1)$ and Adult $(n=16) . * p<0.05$ compared with adults for each molecular species.
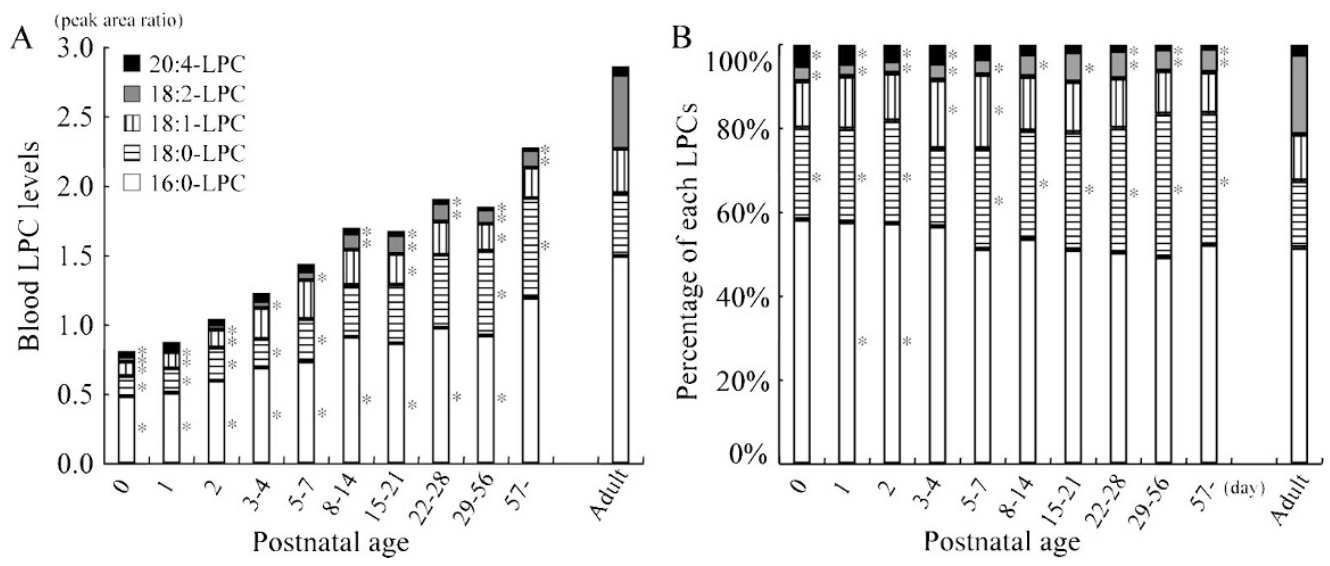

Figure 4. Change in LPCs in VLBW infants: $(A)$ Change in blood LPC levels distinguished by molecular species. LPCs levels were expressed as the peak area ratio of each analyte to the IS (mean). (B) Change in percentage of each LPCs. Postnatal day $0(n=5), 1(n=8), 2(n=$ 8), 3-4 $(n=5), 5-7(n=4), 8-14$ $(n=9), 15-21(n=13), 22-28(n=$ 11), 29-56 $(n=18)$, after $57(n=2)$ and Adult $(n=16)$. $* p<0.05$ compared with adults for each molecular species. after day 8 (Fig. 2). Thus, neonatal blood LPC levels increased each day, and these increases were different between NVLBW and VLBW infants.

Blood LPCs in Neonates. LPCs were then analyzed, because LPCs have been shown to play an important role in infection (9). We recently reported the preliminary data of blood LPCs levels in NVLBW infants (10). In this study, we investigated LPCs levels not only in NVLBW infants, but also VLBW infants at detailed postnatal day points.

Change in blood LPCs levels in NVLBW infants and comparison with those in the adult. The levels of 16:0- and 18:0-LPC were significantly lower than that in adult levels on days $0-4$, and the levels of 18:1-LPC were significantly lower than adult levels on days $0-1$, but increased each day. 16:0and 18:0-LPC levels were not significantly different compared with adult levels after day 5. 18:1-LPC levels were not significantly different compared with adult levels after day 2 . The levels of 18:2-LPC were significantly lower than adult levels during the study period. The levels of 20:4-LPC were significantly higher than adult levels only on day $3-4$. After day 15, statistical analysis was not carried out for all LPC molecular species, because there was only one subject (Fig. 3A).

Change in blood LPCs percentages in NVLBW infants and comparison with those in the adult. The percentage of 16:0-LPC was significantly higher than that in the adult on days 0,1 , and 3-4. The percentages of 18:0- and 18:1-LPC were significantly higher than that in the adult on days $0-14$. The percentage of 18:2-LPC was significantly lower than that in the adult on days $0-14$. The percentage of 20:4-LPC was significantly higher than that in the adult on days $0-7$ (Fig. 3B).

Change in blood LPCs levels in VLBW infants and comparison with those in the adult. The levels of 16:0-LPC were significantly lower than adult levels on days $0-56$. The levels of 18:0-LPC were significantly lower than adult levels until day 7 , but were significantly higher than adult levels after day 29 . The levels of 18:1-LPC were significantly lower than adult levels on days $0-2,15-21$ and 29-56. The levels of 18:2-LPC were significantly lower than adult levels during the study period. The levels of 20:4-LPC were significantly lower than adult levels on day 0 and after day 8 (Fig. 4A).

Change in blood LPCs percentages in VLBW infants and comparison with those in the adult. The percentage of 16:0LPC was significantly higher than that in adults on days 1-2. The percentage of 18:0-LPC was significantly higher than that in the adult during the study period except for on days 3-4. The percentage of 18:1-LPC was significantly higher than that in the adult on days 3-7. The percentage of 18:2-LPC was significantly lower than that in the adult during the study period. The percentage of 20:4-LPC was significantly higher than that in the adult on days 0-4. However, since the percentage of 20:4-LPC decreased gradually, it was significantly lower than that in the adult after day 22 (Fig. 4B).

Comparison of blood LPCs percentages among NVLBW and VLBW infants and the adult. The change in the blood LPCs percentages was similar between NVLBW and VLBW 


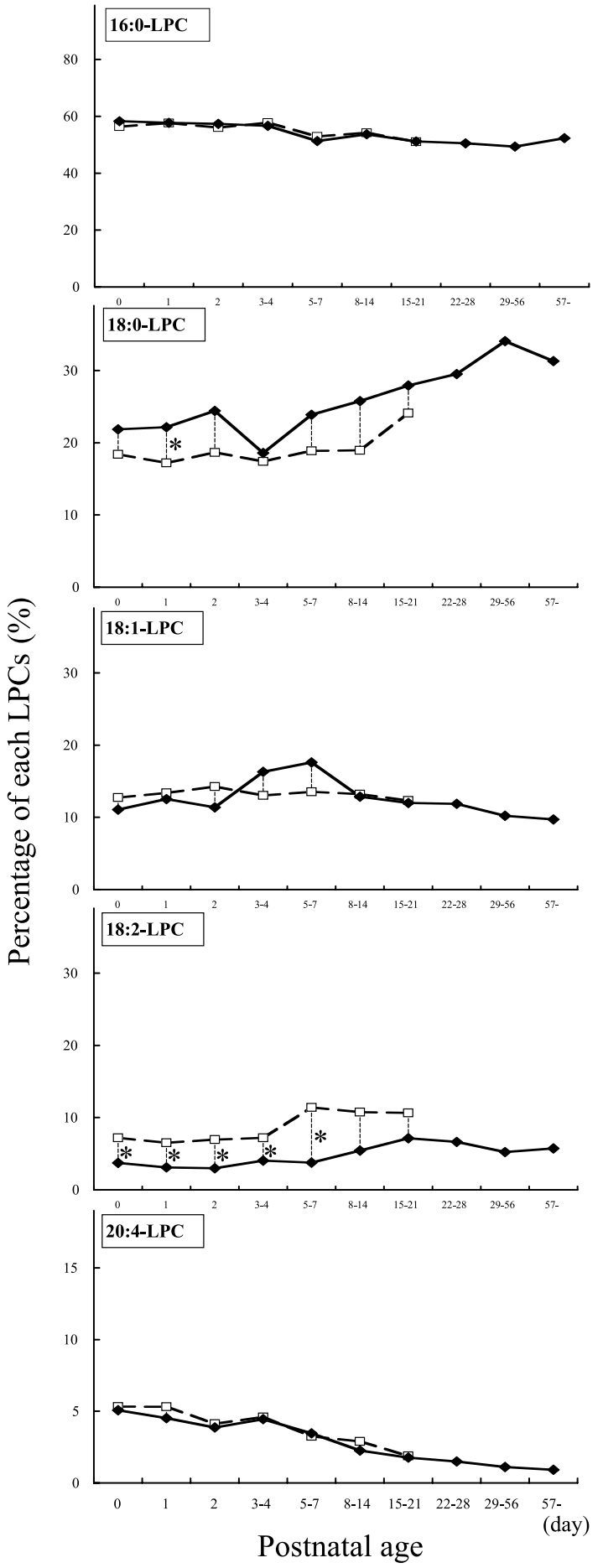

Figure 5. Comparison of blood LPCs (16:0-, 18:0-, 18:1-, 18:2-, and 20:4-LPC) percentages between NVLBW $(\square, n=19)$ and VLBW ( $\bullet$, $n=17$ ) infants: In VLBW infants, the percentage of 18:0-LPC was higher than that in NVLBW infants, and the percentage of 18:2-LPC was lower than that in NVLBW infants. ${ }^{*} p<0.05$.

infants in the 16:0-, 18:1-, and 20:4-LPCs. However, in VLBW infants, the percentage of 18:0-LPC was higher than that in NVLBW infants, and the percentage of 18:2-LPC was lower than that in NVLBW infants, especially on days $0-7$ (Fig. 5).

Changes in the amount of feeding. In VLBW infants, the beginning of feeding was significantly later than that in NV-

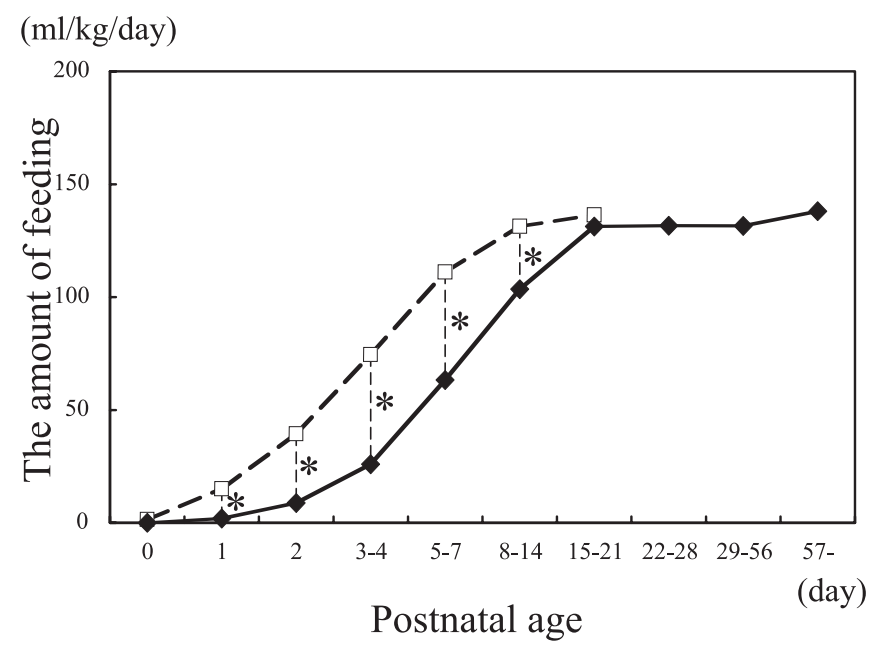

Figure 6. Comparison of the amount of feeding between NVLBW ( $\square, n=$ $19)$ and VLBW ( $\diamond, n=17$ ) infants: In VLBW infants, the increase in the amount of feeding was slower than that in NVLBW infants. Therefore, the amount of feeding in VLBW infants was significantly smaller than that in NVLBW infants on days $1-14$. * $p<0.05$.

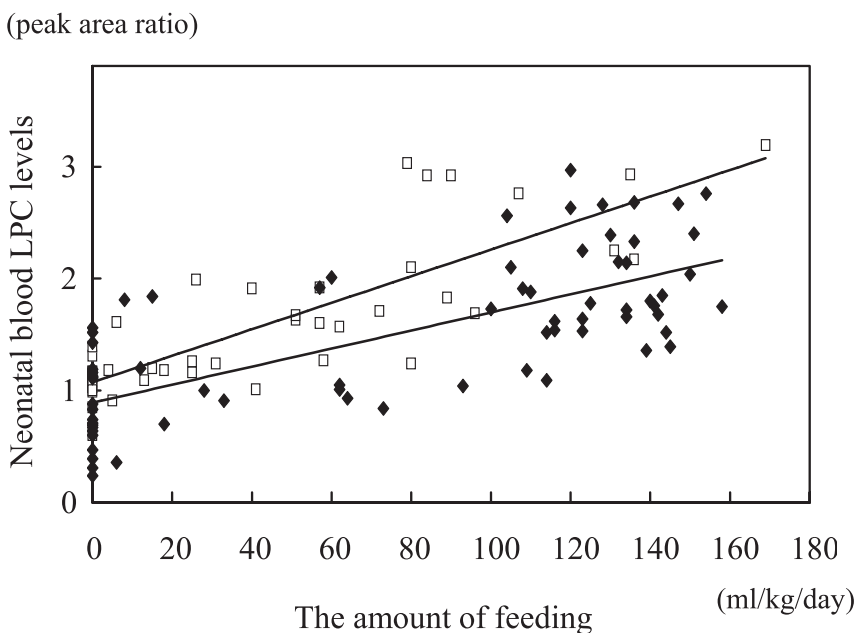

Figure 7. Correlation between neonatal blood LPC levels and the amount of feeding: A statistically significant strong correlation was observed in NVLBW $(\square, p<0.001, r=0.829)$ and VLBW $(\diamond, p<0.001, r=0.722)$ infants.

LBW infants, and age of reached full feeding (over 100 $\mathrm{mL} / \mathrm{kg} / \mathrm{d}$ ) was significantly later than that in NVLBW infants (Table 1). Therefore, in VLBW infants, the increase in the amount of feeding was slower than that in NVLBW infants. Moreover, in VLBW infants, the amount of feeding was significantly smaller than that in NVLBW infants on days 1-14 (Fig. 6).

The relationship between total blood LPC levels and the amount of feeding. A statistically significant strong correlation was observed between total blood LPC levels and the amount of feeding in NVLBW $(p<0.001, r=0.829)$ and VLBW $(p<$ 0.001, $r=0.722$ ) infants (Fig. 7).

\section{DISCUSSION}

We recently reported blood LPC levels only at day $0-1$ and 4-8 in NVLBW infants (10). In this current paper, the analyzed subject was expanded to VLBW infants. Moreover, 
blood LPCs levels were measured at the detailed postnatal day points. In this study, total blood LPC levels at birth were significantly lower than those in the adult. LPC levels increased gradually after birth, and then total blood LPC levels reached adult levels. However, a different change was shown between NVLBW and VLBW infants, and this difference was similar to the difference in the course of the feeding amount between these two groups. LPC is found in human breast milk (11), and in this study, a strong positive correlation was observed between neonatal blood LPC levels and the amount of feeding. These results suggest that the amount of feeding influences the increase in neonatal blood LPC levels.

In NVLBW infants, the total blood LPC levels at days 3-4 had reached adult levels. However, in VLBW infants, blood LPC levels increased slowly and reached adult levels after day 57, and total blood LPC levels in VLBW infants were significantly lower than those in NVLBW infants on days 2-7. The amount of feeding in VLBW infants was significantly smaller than that in NVLBW infants on days 1-14, especially on days 2-7. The factors that influenced an increase in the amount of feeding were the day of beginning of feeding and the day that full feeding was achieved. Feeding begins after respiration and when the circulation is stabilized. In the VLBW infants, because respiration and the circulation were not stabilized soon, the day of the beginning of feeding was late. In addition, since the amount of feeding was carefully increased, the day that full feeding was achieved was later than that in NVLBW infants.

NVLBW infants were classified by birth weight $(\geq 2,500 \mathrm{~g}$, and 1,500 2,499 g) and these two groups were compared. In NVLBW infants, a similar pattern was seen in both an increase in blood LPC levels and an increase in the amount of feeding between the neonates $\geq 2,500 \mathrm{~g}$, and 1,500 2,499 g (data not shown). A significant difference in blood LPC levels was observed under a 1,500 g-birth weight. This suggests that not only immature physiologic functions that depend on lowbirth-weight, but also the amount of feeding influence the increase in blood LPC levels in neonates. Because of an increase in blood LPC levels, which depend on an increase in the amount of feeding, the protective system against infection might be improved.

LPC, which stimulates immune cells, is protective against infection (1-7). Newborn infants may be susceptible to infection because low total blood LPC levels cause a decrease in protection against infection. One of the reasons that VLBW infants are susceptible to infection in the long-term may be that they have low total blood LPC levels for longer than NVLBW infants. In our NICU, all infectious diseases occurred in limited postnatal days when total blood LPC levels were significantly lower than adult levels. In NVLBW infants, infectious diseases occurred within postnatal day 2 (day $0 \sim 1$, median: 0 ), and in VLBW infants, they occurred within postnatal day 56 (day $0 \sim 36$, median: 17) (data not shown). Accordingly, we believe that neonatal blood LPC levels are an important parameter, which show neonatal immune function.

The specific compositions of LPCs found in neonates were compared with the adult as follows. Percentages of 16:0-, 18:0- and 18:1-LPC in neonates were higher than those in the adult, and the percentage of 18:2-LPC was lower than that in the adult. The percentage of 20:4-LPC was higher than that in the adult in the early postnatal age, but this percentage subsequently decreased and thus it was lower than that in the adult. These findings may be associated with the difference in immunity between neonates and adults.

Recent research has shown that 18:0- and 18:1-LPC have a therapeutic effect against sepsis in the mouse model, and these two LPCs are more effective than another LPCs (9). Consequently, we investigated the main LPCs. In both NVLBW and VLBW infants, the sum percentage of 18:0and 18:1-LPC was significantly higher than that in the adult, at all ages. Newborn infants may enhance protection against infection by raising the sum percentage of 18:0- and 18:1-LPC under the condition that total blood LPC levels are low.

In VLBW infants, each blood LPCs increased each day, especially 18:0-LPC. In addition, the percentage of 18:0-LPC increased each day. The percentage of 18:0-LPC on days 29-56 was significantly higher than that on day 0. In VLBW infants, the percentage of 18:0-LPC was higher than that in NVLBW infants. The VLBW infants may enhance the protection against infection by raising the percentage of 18:0LPC under the condition that total blood LPC levels are lower than those in NVLBW infants.

With regard to the other LPCs, the percentage of 16:0-LPC was not significantly different in NVLBW and VLBW infants. In VLBW infants, the percentage of 18:2-LPC was considerably lower than that in the adult. The percentage of 18:2-LPC in VLBW infants was lower than that in NVLBW infants. In VLBW infants, the percentage of 20:4-LPC was higher than that in the adult until day 7, but it decreased each day after birth. Moreover, the percentage of 20:4-LPC on days 8-56 was significantly lower than that on day 0 .

Preventative strategies against infection are very important. Early and accurate diagnosis and adequate therapies are necessary for protection against infection and prophylaxis is the best strategy. Although various causes of susceptibility to infection have been clarified (12-16), there are few known factors that are related to prophylaxis and treatment, and these prophylaxes and treatments against infection are not effective enough (17-21). Accordingly, there is a need for new methods of reducing risk factors and enhancing the neonate's resistance to infection $(18,19$, 22-24). We suggest that a low level of LPC indicates one of the causes of susceptibility to infection in neonates, especially, VLBW infants, therefore, LPC might be able to be applied for prevention of infection by increasing blood LPC levels as soon as possible after birth.

The neonatal immune system is currently not well understood. The present research has shown blood LPCs characteristics in neonates. This study is a fundamental investigation for examining the relationship between blood LPCs and infection in neonates, and for applying LPC for a protective strategy against infection.

We conclude that blood LPC levels are low at birth, and that LPC levels in VLBW infants reach adult levels at a later time than those in NVLBW infants. The specific compositions of the 
LPCs are different among adults, NVLBW, and VLBW infants. These findings may be associated with the difference in immunity not only between neonates and adults, but also between NVLBW and VLBW infants.

\section{REFERENCES}

1. Kabarowski JH, Xu Y, Witte ON 2002 Lysophosphatidylcholine as a ligand for immunoregulation. Biochem Pharmacol 64:161-167

2. Coutant F, Perrin-Cocon L, Agaugue S, Delair T, Andre P, Lotteau V 2002 Mature dendritic cell generation promoted by lysophosphatidylcholine. J Immunol 169:1688-1695

3. Ngwenya BZ, Yamamoto N 1986 Effects of inflammation products on immune systems. Lysophosphatidylcholine stimulates macrophages. Cancer Immunol Immunother 21:174-182

4. Gomez-Munoz A, O’Brien L, Hundal R, Steinbrecher UP 1999 Lysophosphatidylcholine stimulates phospholipase D activity in mouse peritoneal macrophages. J Lipid Res 40:988-993

5. Asaoka Y, Oka M, Yoshida K, Sasaki Y, Nishizuka Y 1992 Role of lysophosphatidylcholine in T-lymphocyte activation: involvement of phospholipase A2 in signal transduction through protein kinase C. Proc Natl Acad Sci USA 89:6447-6451

6. Nishi E, Kume N, Ueno Y, Ochi H, Moriwaki H, Kita T 1998 Lysophosphatidylcholine enhances cytokine-induced interferon gamma expression in human $\mathrm{T}$ lymphocytes. Circ Res 83:508-515

7. Silliman CC, Elzi DJ, Ambruso DR, Musters RJ, Hamiel C, Harbeck RJ, Paterson AJ, Bjornsen AJ, Wyman TH, Kelher M, England KM, McLaughlin-Malaxecheberria N, Barnett CC, Aiboshi J, Bannerjee A 2003 Lysophosphatidylcholines prime the NADPH oxidase and stimulate multiple neutrophil functions through changes in cytosolic calcium. J Leukoc Biol 73:511-524

8. Drobnik W, Liebisch G, Audebert FX, Frohlich D, Gluck T, Vogel P, Rothe G, Schmitz G 2003 Plasma ceramide and lysophosphatidylcholine inversely correlate with mortality in sepsis patients. J Lipid Res 44:754-761

9. Yan JJ, Jung JS, Lee JE, Lee J, Huh SO, Kim HS, Jung KC, Cho JY, Nam JS, Suh HW, Kim YH, Song DK 2004 Therapeutic effects of lysophosphatidylcholine in experimental sepsis. Nat Med 10:161-167
10. Takatera A, Takeuchi A, Saiki K, Morisawa T, Yokoyama N, Matsuo M 2006 Quantification of lysophosphatidylcholines and phosphatidylcholines using liquid chromatography-tandem mass spectrometry in neonatal serum. J Chromatogr B Analyt Technol Biomed Life Sci 838:31-36

11. Shoji H, Shimizu T, Kaneko N, Shinohara K, Shiga S, Saito M, Oshida K, Shimizu T, Takase M, Yamashiro Y 2006 Comparison of the phospholipid classes in human milk in Japanese mothers of term and preterm infants. Acta Paediatr 95:996-1000

12. Satwani P, Morris E, Van de Ven C, Cairo MS 2005 Dysregulation of expression of immunoregulatory and cytokine genes and its association with the immaturity in neonatal phagocytic and cellular immunity. Biol Neonate 88:214-227

13. Mussi-Pinhata MM, Rego MA 2005 [Immunological peculiarities of extremely preterm infants: a challenge for the prevention of nosocomial sepsis]. J Pediatr (Rio J) $81:$ S59-S68

14. Levy O 2005 Innate immunity of the human newborn: distinct cytokine responses to LPS and other Toll-like receptor agonists. J Endotoxin Res 11:113-116

15. Adderson EE 2001 Antibody repertoires in infants and adults: effects of T-independent and T-dependent immunizations. Springer Semin Immunopathol 23:387-403

16. Parravicini E, van de Ven C, Anderson L, Cairo MS 2002 Myeloid hematopoietic growth factors and their role in prevention and/or treatment of neonatal sepsis. Transfus Med Rev 16:11-24

17. Carr R, Modi N, Dore C 2003 G-CSF and GM-CSF for treating or preventing neonatal infections. Cochrane Database Syst Rev:CD003066

18. Suri M, Harrison L, Van de Ven C, Cairo MS 2003 Immunotherapy in the prophylaxis and treatment of neonatal sepsis. Curr Opin Pediatr 15:155-160

19. La Gamma EF, De Castro MH 2002 What is the rationale for the use of granulocyte and granulocyte-macrophage colony-stimulating factors in the neonatal intensive care unit? Acta Paediatr Suppl 91:109-116

20. Bernstein HM, Calhoun DA, Christensen RD 2002 Use of myeloid colonystimulating factors in neonates with septicemia. Curr Opin Pediatr 14:91-94

21. Anderson MR, Blumer JL 1997 Advances in the therapy for sepsis in children. Pediatr Clin North Am 44:179-205

22. Bell SG 2006 Immunomodulation, part III: intravenous immunoglobulin. Neonatal Netw 25:213-221

23. Ohlsson A, Lacy JB 2004 Intravenous immunoglobulin for preventing infection in preterm and/or low-birth-weight infants. Cochrane Database Syst Rev:CD000361

24. Baltimore RS 1998 Neonatal nosocomial infections. Semin Perinatol 22:25-32

\section{Erratum}

In the article, "Heat Shock Protein 72 (HSPA1B) Gene Polymorphism and Toll-Like Receptor (TLR) 4 Mutation Are Associated with Increased Risk of Urinary Tract Infection in Children" by Eva Karoly et al. (Pediatr Res 61:371-374), the authors report the following corrections: The affiliation of Attila J. Szabo and Georg S. Reusz is the First Department of Paediatrics at the Semmelweis University of Budapest. The corresponding author is Georg S. Reusz, MD, DsC, First Department of Paediatrics, Semmelweis University, Bókay 53, H-1083 Budapest, Hungary; email: reusz@ gyer1.sote.hu. Both Eva Karoly and Andrea Fekete contributed equally to the study. 